\title{
Eating Disorders and Major Depression: Role of Anger and Personality
}

\section{Abbate-Daga Giovanni, Gramaglia Carla, Marzola Enrica, Amianto Federico, Zuccolin Maria, and Fassino Secondo}

\author{
Eating Disorders Program, Section of Psychiatry, Department of Neuroscience, University of Turin, \\ Via Cherasco 11, 10126 Turin, Italy \\ Correspondence should be addressed to Abbate-Daga Giovanni, giovanni.abbatedaga@unito.it \\ Received 20 April 2011; Accepted 4 August 2011 \\ Academic Editor: C. Robert Cloninger
}

Copyright (๑) 2011 Abbate-Daga Giovanni et al. This is an open access article distributed under the Creative Commons Attribution License, which permits unrestricted use, distribution, and reproduction in any medium, provided the original work is properly cited.

This study aimed to evaluate comorbidity for MD in a large ED sample and both personality and anger as clinical characteristics of patients with ED and MD. We assessed $838 \mathrm{ED}$ patients with psychiatric evaluations and psychometric questionnaires: Temperament and Character Inventory, Eating Disorder Inventory-2, Beck Depression Inventory, and State-Trait Anger Expression Inventory. $19.5 \%$ of ED patients were found to suffer from comorbid MD and $48.7 \%$ reported clinically significant depressive symptomatology: patients with Anorexia Binge-Purging and Bulimia Nervosa were more likely to be diagnosed with MD. Irritable mood was found in the $73 \%$ of patients with MD. High Harm Avoidance (HA) and low Self-Directedness (SD) predicted MD independently of severity of the ED symptomatology, several clinical variables, and ED diagnosis. Assessing both personality and depressive symptoms could be useful to provide effective treatments. Longitudinal studies are needed to investigate the pathogenetic role of HA and SD for ED and MD.

\section{Introduction}

Lifetime comorbidity between Eating Disorders (EDs) and Mood Disorders has been confirmed by several retrospective studies reporting that in Anorexia Nervosa (AN) the prevalence of mood disorders varies between $64.1 \%$ and $96 \%$ whereas in Bulimia Nervosa (BN) between 50\% and $90 \%$. In addition, a substantial part of individuals affected by an ED is likely to be affected also by a mood disorder, and the current comorbidity varies from 12.7 to $68 \%$ in $\mathrm{AN}$ and is about $40 \%$ in $\mathrm{BN}$ [1]. Major Depression (MD) is the most prevalent comorbid mood disorder in ED patients, and the severity of depressive symptomatology seems to be related to the ED one [2-5].

In spite of the importance of this topic, most of previous studies on mood in ED were conducted on small samples (e.g., fewer than 30 cases), and the role of age, duration of illness, and weight were not considered. Moreover, ED subtypes and their differences were not carefully classified, particularly Eating Disorder Not Otherwise Specified
(EDNOS) [6], and the dimensional assessment of depressive symptomatology wasnot evaluated in detail. Furthermore, the experience and expression of anger in patients with comorbid depression and ED have been relatively neglected, even though hostility and aggressiveness are commonly reported in ED populations $[7,8]$.

Indeed anxious/preoccupied behaviors, mood intolerance, and dysthymic traits have been reported in ED patients $[5,9,10]$. Studies conducted with the Temperament and Character Inventory (TCI) [11] have found that ED individuals both in the acute phase $[9,12,13]$ and after remission [14-16] performed higher scores of Harm Avoidance (HA) and low scores of Self-Directedness (SD) than healthy controls. Individuals with these personality features are thought to have poorer abilities to cope with stressful life events $[9,13]$ and, although future studies are needed high HA and low SD have been proposed as potential risk factors for ED and not only consequences of the illness [13]. Various authors have found such alterations of personality dimensions - high HA and low SD-in patients with MD 
[17-19] also after remission [20-22]: hence, it should be noted that the alterations of these traits are not only state dependent, as suggested by some studies [23]. Despite these findings, few studies have examined the personality traits of patients with comorbid $\mathrm{ED}$ and $\mathrm{MD}$, after controlling for eating psychopathology and other clinical variables.

With this study we aimed to (a) evaluate the prevalence of a current MD in a large sample of ED patients; (b) assess the prevalence of MD with irritable mood in ED patients; (c) provide data supporting the correlation between MD and ED severity; (d) show possible differences between ED patients with and without MD, independently from the severity of eating symptomatology.

\section{Materials and Methods}

The sample consisted of 838 patients admitted to the outpatient service of the ED Program of the University of Turin between the 1st of January 2003 and the 31st of December 2010. All subjects were diagnosed with an ED, and the sample was represented by the following subjects: $\mathrm{AN}$, restricting type (AN-R), $n=214$; AN, binge-purging type (AN-BP), $n=103 ; \mathrm{BN}$, purging type (BN-E), $n=$ 223, Eating Disorder Not Otherwise Specified (EDNOS), $n=298$. Patients with BN, nonpurging type, were excluded because their number $(n=13)$ was not statistically relevant. Diagnoses of ED and MD were based on the structured clinical interview for DSM-IV (SCID-I) [24]. Exclusion criteria were medical comorbidity (e.g., epilepsy or diabetes), drug abuse, and male gender.

The first two assessment interviews were conducted by psychiatrists experienced in the diagnosis and treatment of ED. Irritable mood and angry outbursts were assessed according to the criteria proposed by Fava and Kellner [25] and evaluated with clinical interviews derived by authors' questionnaires. Patients completed the selfreport questionnaires described below between the first and the second interview. After complete description of the study to the subjects, written informed consent was obtained. The Italian version of self-rating instruments was used.

2.1. Temperament and Character Inventory (TCI). The TCI [11] is divided into seven dimensions. Four of these assess temperament (Novelty Seeking [NS], Harm Avoidance [HA], Reward Dependence [RD], and Persistence $[\mathrm{P}]$ ), defined as partly heritable emotional responses, stable throughout life, mediated by neurotransmitters in the central nervous system. The other three dimensions assess character (Self-Directedness [SD], Cooperativeness [C], and SelfTranscendence $[\mathrm{ST}]$ ), defined as the overall personality traits acquired through experience.

2.2. State-Trait Anger Expression Inventory (STAXI). The 44item STAXI [26] measures the intensity of anger as an emotional state (State-anger) and the disposition toward anger as a personality trait (Trait-anger). Anger ExpressionIn (AX-In) measures the suppression of angry feelings. Anger
Expression-Out (AX-Out) measures the frequency of the expression of anger toward other people or objects in the environment. Anger Expression Control (AX-Con) measures the control of anger. AX/Ex provides a general index of the expression of anger.

2.3. Beck Depression Inventory (BDI). The BDI [27] is a selfreport questionnaire used to assess the severity of symptoms of depression. Clinical euthymia is defined by scores lower than 10. The BDI has been found to be a reliable instrument for assessing depressive symptoms in ED patients.

2.4. Eating Disorder Inventory-2 (EDI-2). The EDI-2 [28] is a self-report measure of disordered eating attitudes and behaviors, as well as of personality traits common to individuals with ED. Eleven subscales evaluate symptoms and psychological correlates of ED.

2.5. Statistical Analysis. Statistical analyses were carried out using Statistical Package for Social Sciences (SPSS) software version 13.0 for Windows (SPSS 13.0 Application Guide. Chicago, SPSS, Inc., 2004). Categorical data were compared using the chi-squared test, and continuous data were analysed using a two-tailed independent $t$-test. Age, age of onset of the disorder, duration of illness, and Body Mass Index (BMI) were analysed in terms of confounding variables using a Univariate General Linear Model.

A logistic regression analysis was performed to detect personality variables that independently relate with MD. The presence/absence of MD was regarded as a dependent variable. ED diagnosis, duration of illness expressed in months, $\mathrm{BMI}$, age, age of onset of the disorder, presence/absence of irritable mood, and scores on the TCI, EDI-2, and STAXI scales were included as independent variables.

To assess the possible correlation with the depressive state of personality traits we found as significant at the linear regression has been checked the linear correlation (Pearson bivariate) between BDI and personality score and we performed also a MANOVA with personality scores as dependent variables, depressive versus nondepressive group as fixed factor, the BDI score as covariate, an the BDI group interaction.

\section{Results}

3.1. Sociodemographic and Clinical Features of the Sample. Sociodemographic and clinical features are reported in Tables 1 and 2 .

3.2. MD Diagnosis and Depressive Symptomatology. Subjects with MD represent the $19.5 \%(n=161)$ of the sample: $15.3 \%$ of AN-R $(n=33), 25.5 \%$ of AN-BP $(n=25), 25.3 \%$ of $\mathrm{BN}(N=56)$, and $16 \%$ of EDNOS $(n=47)$. Significant differences were found among AN and EDNOS individuals and the other ED subtypes $\left(\chi^{2}=11.752 ; P=0.008\right)$.

Patients with MD did not show any significant difference when compared to those without $\mathrm{MD}$ in regard to age, age of onset of the disorder, duration of illness, and BMI 
TABLE 1: Sociodemographic characteristics of the sample.

\begin{tabular}{lc}
\hline & Total sample $(n=838)$ \\
\hline Female & $100 \%$ \\
Caucasian & $100 \%$ \\
\hline
\end{tabular}

TABLE 2: Clinical features of the sample.

\begin{tabular}{lcccc}
\hline & ED without MD & ED with MD & $t$ & $P$ \\
\hline Age & $28.54 \pm 9.36$ & $29.68 \pm 10.08$ & -1.376 & 0.169 \\
$\begin{array}{l}\text { Age of onset } \\
\text { Duration of }\end{array}$ & $19.93 \pm 7.85$ & $20.24 \pm 8.18$ & -0.450 & 0.653 \\
$\begin{array}{l}\text { illness } \\
\text { (months) }\end{array}$ & $103.49 \pm 94.90$ & $113.66 \pm 103.03$ & -1.207 & 0.228 \\
$\begin{array}{l}\text { BMI: total } \\
\text { group }\end{array}$ & $18.88 \pm 3.72$ & $19.33 \pm 3.72$ & -1.394 & 0.164 \\
\hline
\end{tabular}

ED: Eating Disorder; MD: Major Depression; BMI: body mass index.

(see Table 2). The BDI scores of subjects with MD were significantly different from those without this diagnosis $(37.1 \pm 4.5$ versus $11.1 \pm 4.8 ; F=550.5 ; P=0.001)$, after controlling for age, age of onset of the disorder, duration of illness, and BMI.

The BDI scores of 408 patients ( $48.7 \%$ of the sample) who were not diagnosed with MD were higher than 10 and so clinically significant; there were statistically significant differences among diagnostic subtypes in this regard $\left(\chi^{2}=\right.$ 9.3859; $P=0.02$ ). Considering both the $48.7 \%$ of the sample with a BDI score $>10$ and the $19.5 \%$ of MD patients the total percentage of patients with relevant depressive symptomatology is $68.2 \%$.

Patients with MD, irritable mood, anger attacks, or angry outbursts made up $73 \%$ of the sample, with no significant differences among diagnostic groups $\left(\chi^{2}=\right.$ 1.321; $P=0.724)$. Moreover, subjects with MD obtained more pathological scores on all STAXI subscales, even after controlling for age, age of onset of the disorder, duration of illness, and BMI, than did patients without MD diagnosis (Table 3). Also subject with clinically significant depressive symptoms $($ BDI > 10) reported higher STAXI scores than patients without such symptomatology (data not shown).

3.3. Eating Psychopathology. After controlling for age, age of onset of the disorder, duration of illness, and BMI, patients with MD showed higher scores on all EDI-2 scales than did those without this diagnosis (Table 4).

3.4. Personality. MD patients performed higher scores than those without MD on the HA scale and lower scores on the $\mathrm{RD}, \mathrm{SD}$, and $\mathrm{C}$ scales of the TCI, even after controlling for age, age of onset of the disorder, duration of illness, and BMI (Table 5).

3.5. Logistic Regression. The logistic regression model was significant $\left(\chi^{2}=212.7\right.$; df: $36 ; P<0.001 ; R$-square $\left.=0.454\right)$. The state anger STAXI subscale $(B=0.086$; Wald $=13.315$;
TABle 3: State-Trait Anger Expression Inventory (STAXI).

\begin{tabular}{lcccc}
\hline & ED without MD & ED with MD & $t$ & $P$ \\
\hline S-Anger & $13.85 \pm 5.53$ & $20.50 \pm 8.99$ & -11.394 & 0.001 \\
T-Anger & $22.14 \pm 6.56$ & $25.91 \pm 6.46$ & -6.469 & 0.001 \\
T-Anger/T & $8.00 \pm 3.17$ & $9.57 \pm 3.41$ & -5.323 & 0.001 \\
T-Anger/R & $10.37 \pm 4.04$ & $11.85 \pm 2.88$ & -4.191 & 0.001 \\
AX-In & $18.90 \pm 5.70$ & $22.21 \pm 4.98$ & -6.508 & 0.001 \\
AX-Out & $16.02 \pm 5.08$ & $17.66 \pm 5.48$ & -3.494 & 0.001 \\
AX-Con & $20.51 \pm 6.01$ & $18.10 \pm 6.40$ & 4.330 & 0.001 \\
AX-Ex & $30.40 \pm 11.33$ & $37.57 \pm 10.57$ & -7.020 & 0.001 \\
\hline
\end{tabular}

ED: Eating Disorder; MD: Major Depression; S-Anger: State-anger; T-Anger: Trait-anger; AX-In: Anger Expression-In; AX-Out: Anger Expression-Out; AX-Con: Anger Expression Control; AX-Ex: Anger Expression.

TABLE 4: Eating Disorder Inventory-2 (EDI-2).

\begin{tabular}{lcccc}
\hline & ED without MD & ED with MD & $t$ & $P$ \\
\hline DT & $11.24 \pm 7.27$ & $15.83 \pm 6.29$ & -7.400 & 0.001 \\
B & $5.91 \pm 5.56$ & $8.49 \pm 6.38$ & -5.138 & 0.001 \\
BD & $12.33 \pm 7.72$ & $18.04 \pm 6.71$ & -8.657 & 0.001 \\
I & $8.33 \pm 6.37$ & $17.46 \pm 6.88$ & -16.125 & 0.001 \\
P & $5.25 \pm 4.13$ & $7.02 \pm 4.45$ & -4.834 & 0.001 \\
ID & $5.57 \pm 4.46$ & $9.30 \pm 4.87$ & -9.359 & 0.001 \\
IA & $9.16 \pm 6.60$ & $15.54 \pm 7.33$ & -10.802 & 0.001 \\
MF & $6.42 \pm 5.03$ & $8.95 \pm 6.03$ & -5.514 & 0.001 \\
A & $6.69 \pm 4.25$ & $9.91 \pm 4.60$ & -8.517 & 0.001 \\
IR & $6.43 \pm 5.75$ & $12.59 \pm 6.76$ & -11.822 & 0.001 \\
SI & $7.10 \pm 5.03$ & $12.00 \pm 4.38$ & -11.399 & 0.001 \\
\hline
\end{tabular}

ED: Eating Disorder; MD: Major Depression; DT: drive for thinness; B: bulimia; BD: body dissatisfaction; I: Ineffectiveness; P: perfectionism; ID: interpersonal distrust; IA: interoceptive awareness; MF: maturity fears; A: Asceticism; IR: impulse regulation; SI: social insecurity.

TABLE 5: Temperament and Character Inventory (TCI).

\begin{tabular}{lcccc}
\hline & ED without MD & ED with MD & $t$ & $P$ \\
\hline NS & $20.70 \pm 9.75$ & $19.53 \pm 6.34$ & 1.450 & 0.148 \\
HA & $22.00 \pm 9.35$ & $26.88 \pm 5.69$ & -6.329 & $\mathbf{0 . 0 0 1}$ \\
RD & $15.53 \pm 5.50$ & $14.31 \pm 3.65$ & 2.675 & $\mathbf{0 . 0 0 8}$ \\
P & $5.28 \pm 5.14$ & $4.66 \pm 2.03$ & 1.498 & 0.134 \\
SD & $23.08 \pm 8.30$ & $15.38 \pm 6.29$ & 10.983 & $\mathbf{0 . 0 0 1}$ \\
C & $30.75 \pm 7.34$ & $27.10 \pm 7.50$ & 5.630 & $\mathbf{0 . 0 0 1}$ \\
ST & $13.86 \pm 7.45$ & $13.85 \pm 6.72$ & 0.002 & 0.998 \\
\hline
\end{tabular}

ED: Eating Disorder; MD: Major Depression; NS: novelty seeking; HA: harm avoidance; RD: reward dependence; P: persistence; SD: Self-Directedness; C: cooperativeness; ST: Self-transcendence.

$P<0.001)$, the HA subscale of the TCI $(B=0.05$; Wald $=$ 5.85; $P<0.016)$, the SD subscale of the TCI $(B=0.074$; Wald $=8.015 ; P<0.005)$, and Ineffectiveness as measured by the EDI-2 $(B=0.064$; Wald $=5.466 ; P=0.019)$ 
independently correlated with MD. Age, age of onset, ED diagnosis, BMI, episodes of binge-eating and vomiting per week, irritable mood, and other variables measured by the STAXI, TCI, and EDI-2 were not significant.

3.6. Correlations and MANOVA. BDI scores correlate significantly directly with HA $(r=0.379 ; P<0.001)$ and inversely with $(r=-0,589 P<0.001)$. Using the MANOVA, HA, and SD differences remain significant even when controlled for BDI scores and for the interaction BDI group (HA: $F=$ 75.031; $P<0.001$; SD: $F=227.362 ; P<0.001)$. Also the BDI score effect was found significant for both variables (both variables: $P<0.001)$.

\section{Discussion}

4.1. Characteristics of Depressive Symptomatology. Data from the present study reported lower MD rates than other studies; such a difference could be due to participants' different stages of illness and it should be also noted that we considered only outpatients while other studies included inpatients.

Significant differences were demonstrated among diagnostic subtypes; patients with purging behaviours (AN-BP and $\mathrm{BN}$ ) were more likely to be diagnosed with $\mathrm{MD}$ when compared to AN and EDNOS. This association is supported by previous researches showing that individuals with purging symptomatology are more likely to show com-orbid disorders and greater clinical severity [30, 31]. Also our group in previous studies found a correlation-although not related to diagnosis-with purging symptomatology [5].

Moreover, in our sample MD in ED patients seem typically characterized by irritable mood as measured according to Fava and Kellner criteria [25]. To our knowledge, these results have not been described yet in the literature. We found that depressed ED patients were not inhibited or melancholic, but tended to show angry depression, hostility, aggressiveness, anger attacks, and angry outbursts. In fact, irritability and angry outbursts are approximately twice as prevalent among patients with $\mathrm{MD}$ and $\mathrm{ED}$ (73\%) than among depressed patients without ED, as reported in literature $[2,32]$. Results of the STAXI revealed that patients with $\mathrm{MD}$ and $\mathrm{ED}$ experienced greater difficulty in recognizing, managing, and expressing anger than patients without MD. Also logistic regression considered State Anger as one of the four independent variables correlated to MD diagnosis. Anger problems among those with ED have been well documented in the literature $[8,33,34]$, but the role of depressive symptomatology in such difficulties in coping with anger has been rarely considered. Past findings of mood instability deriving from fasting [33], the notorious treatment resistance of ED patients [35], and the presence of selfinjurious behaviours [36] highlight other possible sources for angry outbursts and irritability. However, it should be considered the possibility that anger and oppositionalism can originate from depressive symptoms. The importance of evaluating patients with $\mathrm{AN}$ and $\mathrm{BN}$ for irritable mood is reinforced by the observationthat depression and aggressive- ness totally mediate the connection between ED and suicidal behavior [37]. Given the correlations between depression and anger, the construct of an anxiety/aggression-driven depression has been proposed to correlate depressive and angry aspects, both related to low serotonergic function [38, 39]. It is noteworthy that MD in ED shows some peculiarities since the course is often protracted, the MD recovery may depend on ED type, and antidepressants are not likely to be as effective as in patients with MD without the ED [40]. Dysphoric traits could underlie such differences in features and course of illness [41].

Considering the BDI, the $48.7 \%$ of the sample obtained scores indicating a clinically significant depressive symptomatology (BDI > 10); this datum should be added to the $19.5 \%$ of individuals affected by full MD and therefore the total percentage of individuals with relevant depressive symptoms was $68.2 \%$. Moreover, patients with ED were reported to suffer from a wide spectrum of depressive symptoms [42]. Specific characteristics of MD and such a common depressive symptomatology even not meeting MD full criteria highlight the importance of considering also these psychopathological aspects in assessment, monitoring, and treatment of these disorders.

Moreover, also this lager group of depressed patients reported at the STAXI higher scores than ED patients without depressive symptoms. Therefore, previous considerations regarding the group with both ED and DM about high percentage of irritable mood can be extended to depressed patients without an ED.

4.2. Depressive Symptomatology and Eating Psychopathology. We found that eating psychopathology, as measured by the EDI-2 scales included in this study, was significantly more severe in patients with comorbid ED and MD than in patients with ED without MD. This correlation between a severe depressive symptomatology and ED severity validated the results of previous studies and confirmed expected hypothesis $[2,3,30]$. Moreover it is well known in literature that eating symptomatology is also associated with depression in women, even among those with no history of threshold-level eating disorder symptomatology [43].

The presence of MD represented an index of clinical severity and/or an indication of the acuity of the ED. Therefore, diagnostic evaluation for MD in patients suffering from AN or BN should be considered, and psychotherapeutic involvement in treatment planning should be included as appropriate, also because these patients are often hopeless about the possibility of change and this should be carefully considered in treatments [37]. Indeed, Ametller et al. [44] have demonstrated that high BDI scores at the first psychiatric assessment represent one of the independent predictors of hospitalization.

The logistic regression analysis showed that the Ineffectiveness subscale of EDI-2 independently predicted MD in the sample. Low self-esteem represents the common core symptom of ED and depression. Thus it could be hypothesized that ED treatments based on cognitive-behavioral therapies focused on low self-esteem [45] can be effective for ED depressed subjects. 
Antidepressants might be effective for treating comorbid ED and depression [46]. However, research suggests that psychopharmacological treatment is effective for BN [47], but is of debatable value for $\mathrm{AN}[40,48]$ even to prevent relapse after weight restoration [49].

4.3. Depressive Symptomatology and Personality. Patients with both $\mathrm{ED}$ and $\mathrm{MD}$ were characterized by higher $\mathrm{HA}$ and lower scores on the RD, SD, and C scales of the TCI.

Logistic regression showed that Harm Avoidance and Self-Directedness remained significant after controlling for personal and several clinical variables. These data are consistent with the results of previous studies that have identified these traits as characterizing ED samples when compared to healthy controls [12]. Other studies have shown that these traits persist after recovery from the $\mathrm{ED}[50]$ and that they are altered in adolescents at high risk for developing a clinically significant ED [30]. Both in the acute phase and after remission, also patients with MD but without ED obtained high HA and low SD scores on the TCI [17-23]. In fact, such HA and SD alterations are likely to be both state and trait dependent [51]. Also bipolar euthymic patients showed the same pattern [52]. A recent comprehensive review and meta-analysis of the literature investigated the effects of temperament on vulnerability to depression providing evidence that high HA can be associated both with current depressive symptoms and depressive traits [53]. Interestingly, a significant negative change in HA scores has been reported during treatment, and it can be also related to treatment response and recovery. A minority of studies reported also how low Reward Dependence-another temperamental dimensionwas associated with depressive symptomatology [53].

This study showed that higher HA and low SD scores were correlated with comorbid MD in ED patients; this correlation was found to be independent of the severity of the ED (as measured by BMI, binge-purging behaviours, and EDI-2 scales), age, age of onset, and duration of illness. Other studies have shown that low SD can predict suicide attempts among ED subjects [35, 54].

ED patients with a personality profile characterized by high HA and low RD, SD, and C represent a subgroup of patients likely to experience feelings of inferiority, inadequacy, unhappiness, anxiety, and dependence $[5,31,55-$ 57]. It is well known that ED patients with MD represent a substantial group of patients with specific and semiindependent clinical features and that these features require aimed treatments $[46,58]$.

The cross-sectional design of this study makes it difficult to rule out the possibility that high HA scores represented a risk factor or a "scarring effect" for ED and depression on personality $[18,59]$. Otherwise it is well known the issue of state dependency of HA and SD from depressive disorder [51]. However, there is growing evidence that high Harm Avoidance levels could represent a trait aspect contributing to vulnerability both to ED [41] and mood disorders [53], and in the present study with the MANOVA analysis we found that the BDI score doesnot completely explain the difference in HA and SD scores between depressed and nondepressed groups. Nevertheless, future research is warranted to perform a longitudinal assessment of the general population to compare premorbid personality traits with those associated with both the $\mathrm{ED}$ and depression development during adolescence.

This study is limited by the lack of a control group of healthy subjects or of another clinical population, including patients with other comorbid disorders, and by not considering lifetime comorbidity. On the other hand, one strength of this study is the large sample of patients with MD and ED.

\section{Conclusions}

This study aimed to evaluate comorbidity between ED and $\mathrm{MD}$ and the role of personality as predictor of MD in ED. Our data are in line with previous literature since we found a current prevalence of MD of $19.5 \%$ with significant differences among diagnostic subtypes since patients with purging behaviours were more likely to be affected by $\mathrm{MD}$. Irritability was found to be a feature of MD in ED with rates of irritability and angry outbursts twice as prevalent among patients with MD and ED (73\%) than among depressed patients without ED as reported in the literature. Considering the BDI, the $48.7 \%$ of the sample obtained scores indicating a clinically significant depressive symptomatology (BDI $>10)$. The eating psychopathology, as measured by the EDI-2 scales, was significantly more severe in patients with $\mathrm{MD}$ comorbidity. With regard to personality dimensions, patients with ED and MD showed higher Harm Avoidance and lower scores on the Reward Dependence, Self-Directedness, and Cooperativeness scales of the TCI. The personality dimensions of high HA and low SD could be risk factors in the development of Major Depression in ED individuals because the differences between depressed and non-depressed groups remain significant even after controlling for the BDI score and BDI group interaction.

Clinicians should carefully evaluate in patients with Eating Disorders their depressive symptomatology and the role of anger and personality to provide effective treatments tailored to person and not based only on symptomatology [60].

\section{Acknowledgment}

This study was made possible thanks to a grant from the CRT Company of Turin, Italy, AI/244 19.01.2010 RF = 2009.2734.

\section{References}

[1] N. T. Godart, F. Perdereau, Z. Rein et al., "Comorbidity studies of eating disorders and mood disorders. Critical review of the literature," Journal of Affective Disorders, vol. 97, no. 1-3, pp. 37-49, 2007.

[2] M. Fava, M. Abraham, K. Clancy-Colecchi, J. A. Pava, J. Matthews, and J. F. Rosenbaum, "Eating disorder symptomatology in major depression," Journal of Nervous and Mental Disease, vol. 185, no. 3, pp. 140-144, 1997.

[3] C. Bizeul, J. M. Brun, and D. Rigaud, "Depression influences the EDI scores in anorexia nervosa patients," European Psychiatry, vol. 18, no. 3, pp. 119-123, 2003. 
[4] G. Abbate-Daga, A. Pierò, C. Gramaglia, M. Gandione, and S. Fassino, "An attempt to understand the paradox of anorexia nervosa without drive for thinness," Psychiatry Research, vol. 149, no. 1-3, pp. 215-221, 2007.

[5] G. A. Daga, C. Gramaglia, U. Bailer, S. Bergese, E. Marzola, and S. Fassino, "Major Depression and avoidant personality traits in Eating Disorders," Psychotherapy and Psychosomatics, vol. 80, pp. 319-320, 2011.

[6] C. G. Fairburn and P. J. Harrison, "Eating disorders," Lancet, vol. 361, no. 9355, pp. 407-416, 2003.

[7] S. Fassino, G. A. Daga, A. Pierò, P. Leombruni, and G. G. Rovera, "Anger and personality in eating disorders," Journal of Psychosomatic Research, vol. 51, no. 6, pp. 757-764, 2001.

[8] E. Truglia, E. Mannucci, S. Lassi, C. M. Rotella, C. Faravelli, and V. Ricca, "Aggressiveness, anger and eating disorders: a review," Psychopathology, vol. 39, no. 2, pp. 55-68, 2006.

[9] S. Fassino, F. Amianto, C. Gramaglia, F. Facchini, and G. Abbate Daga, "Temperament and character in eating disorders: ten years of studies," Eating and Weight Disorders, vol. 9, no. 2, pp. 81-90, 2004.

[10] L. R. Lilenfeld, S. Wonderlich, L. P. Riso, R. Crosby, and J. Mitchell, "Eating disorders and personality: a methodological and empirical review," Clinical Psychology Review, vol. 26, no. 3, pp. 299-320, 2006.

[11] C. R. Cloninger, D. M. Svrakic, and T. R. Przybeck, "A psychobiological model of temperament and character," Archives of General Psychiatry, vol. 50, no. 12, pp. 975-990, 1993.

[12] S. Fassino, G. Abbate-Daga, F. Amianto, P. Leombruni, S. Boggio, and G. G. Rovera, "Temperament and character profile of eating disorders: a controlled study with the temperament and character inventory," International Journal of Eating Disorders, vol. 32, no. 4, pp. 412-425, 2002.

[13] L. R. Lilenfeld, "Personality and temperament," Current Topics in Behavioral Neurosciences, vol. 6, pp. 3-16, 2011.

[14] C. M. Bulik, P. F. Sullivan, J. L. Fear, and A. Pickering, "Outcome of anorexia nervosa: eating attitudes, personality, and parental bonding," International Journal of Eating Disorders, vol. 28 , no. 2 , pp. 139-147, 2000.

[15] K. L. Klump, M. Strober, C. M. Bulik et al., "Personality characteristics of women before and after recovery from an eating disorder," Psychological Medicine, vol. 34, no. 8, pp. 1407-1418, 2004.

[16] H. Bloks, H. W. Hoek, I. Callewaert, and E. van Furth, "Stability of personality traits in patients who received intensive treatment for a severe eating disorder," Journal of Nervous and Mental Disease, vol. 192, no. 2, pp. 129-138, 2004.

[17] R. T. Joffe, R. M. Bagby, A. J. Levitt, J. J. Regan, and J. D. Parker, "The Tridimensional Personality Questionnaire in major depression," American Journal of Psychiatry, vol. 150, no. 6, pp. 959-960, 1993.

[18] S. Hirano, T. Sato, T. Narita et al., "Evaluating the state dependency of the Temperament and Character Inventory dimensions in patients with major depression: a methodological contribution," Journal of Affective Disorders, vol. 69, no. 1-3, pp. 31-38, 2002.

[19] A. Farmer, A. Mahmood, K. Redman, T. Harris, S. Sadler, and P. McGuffin, "A sib-pair study of the temperament and character inventory scales in major depression," Archives of General Psychiatry, vol. 60, no. 5, pp. 490-496, 2003.

[20] J. Richter, M. Eisemann, and G. Richter, "Temperament and character during the course of unipolar depression among inpatients," European Archives of Psychiatry and Clinical Neuroscience, vol. 250, no. 1, pp. 40-47, 2000.
[21] K. Y. Abrams, S. K. Yune, S. J. Kim et al., "Trait and state aspects of harm avoidance and its implication for treatment in major depressive disorder, dysthymic disorder, and depressive personality disorder," Psychiatry and Clinical Neurosciences, vol. 58, no. 3, pp. 240-248, 2004.

[22] D. J. Smith, L. Duffy, M. E. Stewart, W. J. Muir, and D. H. Blackwood, "High harm avoidance and low selfdirectedness in euthymic young adults with recurrent, earlyonset depression," Journal of Affective Disorders, vol. 87, no. 1, pp. 83-89, 2005.

[23] R. F. P. de Winter, R. Wolterbeek, P. Spinhoven, F. G. Zitman, and L. G. Goekoop, "Character and temperament in major depressive disorder and a highly anxious-retarded subtype derived from melancholia," Comprehensive Psychiatry, vol. 48, no. 5, pp. 426-435, 2007.

[24] M. B. First, R. L. Spitzer, M. Gibbon, and J. B. W. Williams, Structured Clinical Interview for DSM-IV (SCID-I) (User's Guide and Interview). Clinical Version, Biometrics Research Department, New York, NY, USA, 1997.

[25] G. A. Fava and R. Kellner, "Prodromal symptoms in affective disorders," American Journal of Psychiatry, vol. 148, no. 7, pp. 823-830, 1991.

[26] C. D. Spielberg, State-Trait Anger Expression Inventory: Professional Manual, Psychological Assessment Resources, Odessa, NY, USA, 1996.

[27] A. T. Beck, C. H. Ward, M. Mendelson, J. Mock, and J. Erbaugh, "An inventory for measuring depression," Archives of General Psychiatry, vol. 4, pp. 561-571, 1961.

[28] D. Garner, EDI-2. Eating Disorder Inventory-2. Professional Manual, Psychological Assessment Resources, Odessa, NY, USA, 1991.

[29] J. Dowson, "Associations of the severity of depressive disorders in women with psychogenic low weight," Journal of Affective Disorders, vol. 78, no. 3, pp. 279-284, 2004.

[30] G. Abbate-Daga, C. Gramaglia, G. Malfi, A. Pierò, and S. Fassino, "Eating problems and personality traits. An Italian pilot study among 992 high school students," European Eating Disorders Review, vol. 15, no. 6, pp. 471-478, 2007.

[31] D. Westen and J. Harnden-Fischer, "Personality profiles in eating disorders: rethinking the distinction between axis I and axis II," American Journal of Psychiatry, vol. 158, no. 4, pp. 547562, 2001.

[32] G. A. Fava, C. Ruini, and C. Belaise, "The concept of recovery in major depression," Psychological Medicine, vol. 37, no. 3, pp. 307-317, 2007.

[33] S. Fassino, G. A. Daga, A. Pierò, P. Leombruni, and G. G. Rovera, "Anger and personality in eating disorders," Journal of Psychosomatic Research, vol. 51, no. 6, pp. 757-764, 2001.

[34] S. Fassino, G. Abbate-Daga, A. Pierò, P. Leombruni, and G. G. Rovera, "Dropout from brief psychotherapy within a combination treatment in bulimia nervosa: role of personality and anger," Psychotherapy and Psychosomatics, vol. 72, no. 4, pp. 203-210, 2003.

[35] A. S. Kaplan, "Psychological treatments for anorexia nervosa: a review of published studies and promising new directions," Canadian Journal of Psychiatry, vol. 47, no. 3, pp. 235-242, 2002.

[36] A. Favaro, P. Santonastaso, P. Monteleone et al., "Self-injurious behavior and attempted suicide in purging bulimia nervosa: associations with psychiatric comorbidity," Journal of Affective Disorders, vol. 105, no. 1-3, pp. 285-289, 2008.

[37] P. Miotto and A. Preti, "Eating disorders and suicide ideation: the mediating role of depression and aggressiveness," Comprehensive Psychiatry, vol. 48, no. 3, pp. 218-224, 2007. 
[38] H. M. van Praag, "Anxiety/aggression-driven depression. A paradigm of functionalization and verticalization of psychiatric diagnosis," Progress in Neuro-Psychopharmacology and Biological Psychiatry, vol. 25, pp. 893-924, 2001.

[39] H. M. van Praag, "Serotonin disturbances and suicide risk: is aggression or anxiety the interjacent link?" Crisis, vol. 21, no. 4, pp. 160-162, 2000.

[40] D. Mischoulon, K. T. Eddy, A. Keshaviah et al., "Depression and eating disorders: treatment and course," Journal of Affective Disorders, vol. 130, no. 3, pp. 470-477, 2011.

[41] W. H. Kaye, J. L. Fudge, and M. Paulus, "New insights into symptoms and neurocircuit function of anorexia nervosa," Nature Reviews Neuroscience, vol. 10, no. 8, pp. 573-584, 2009.

[42] R. C. Casper, "Depression and eating disorders," Depression and Anxiety, vol. 8, no. 1, pp. 96-104, 1998.

[43] J. E. Wildes, A. D. Simons, and M. D. Marcus, "Bulimic symptoms, cognitions, and body dissatisfaction in women with major depressive disorder," International Journal of Eating Disorders, vol. 38, no. 1, pp. 9-17, 2005.

[44] L. Ametller, J. Castro, E. Serrano, E. Martínez, and J. Toro, "Readiness to recover in adolescent anorexia nervosa: prediction of hospital admission," Journal of Child Psychology and Psychiatry and Allied Disciplines, vol. 46, no. 4, pp. 394400, 2005.

[45] G. Waller, E. Corstorphine, and V. Mountford, "The role of emotional abuse in the eating disorders: implications for treatment," Eating Disorders, vol. 15, no. 4, pp. 317-331, 2007.

[46] J. K. Salminen, H. Karlsson, J. Hietala et al., "Short-term psychodynamic psychotherapy and fluoxetine in major depressive disorder: a randomized comparative study," Psychotherapy and Psychosomatics, vol. 77, no. 6, pp. 351-357, 2008.

[47] K. J. Pederson, J. L. Roerig, and J. E. Mitchell, "Towards the pharmacotherapy of eating disorders," Expert Opinion on Pharmacotherapy, vol. 4, no. 10, pp. 1659-1678, 2003.

[48] G. Abbate Daga, L. Gianotti, V. Mondelli, R. Quartesan, and S. Fassino, "The psychopharmacotherapy of anorexia nervosa: clinical, neuroendocrine and metabolic aspects," Journal of Endocrinological Investigation, vol. 27, no. 10, pp. 975-981, 2004.

[49] B. T. Walsh, A. S. Kaplan, E. Attia et al., "Fluoxetine after weight restoration in anorexia nervosa: a randomized controlled trial," JAMA, vol. 295, no. 22, pp. 2605-2612, 2006.

[50] K. L. Klump, C. M. Bulik, C. Pollice et al., "Temperament and character in women with anorexia nervosa," Journal of Nervous and Mental Disease, vol. 188, no. 9, pp. 559-567, 2000.

[51] S. Hirano, T. Sato, T. Narita et al., "Evaluating the state dependency of the Temperament and Character Inventory dimensions in patients with major depression: a methodological contribution," Journal of Affective Disorders, vol. 69, no. 1-3, pp. 31-38, 2002.

[52] C. Engström, S. Brändström, S. Sigvardsson, R. Cloninger, and P. O. Nylander, "Bipolar disorder: I. Temperament and character," Journal of Affective Disorders, vol. 82, no. 1, pp. 131-134, 2004.

[53] O. Kampman and O. Poutanen, "Can onset and recovery in depression be predicted by temperament? A systematic review and meta-analysis," Journal of Affective Disorders. In press.

[54] C. M. Bulik, P. F. Sullivan, and P. R. Joyce, "Temperament, character and suicide attempts in anorexia nervosa, buli-mia nervosa and major depression," Acta Psychiatrica Scandinavica, vol. 100, no. 1, pp. 27-32, 1999.
[55] D. Westen and S. Muderrisoglu, "Assessing personality disorders using a systematic clinical interview: evaluation of an alternative to structured interviews," Journal of Personality Disorders, vol. 17, no. 4, pp. 351-369, 2003.

[56] H. Thompson-Brenner, K. T. Eddy, D. A. Satir, C. L. Boisseau, and D. Westen, "Personality subtypes in adolescents with eating disorders: validation of a classification approach," Journal of Child Psychology and Psychiatry, vol. 49, no. 2, pp. 170-180, 2008.

[57] H. Thompson-Brenner and D. Westen, "Personality subtypes in eating disorders: validation of a classification in a naturalistic sample," British Journal of Psychiatry, vol. 186, pp. 516-524, 2005.

[58] H. L. Van, J. Dekker, J. Peen, G. van Aalst, and R. A. Schoevers, "Identifying patients at risk of complete nonresponse in the outpatient treatment of depression," Psychotherapy and Psychosomatics, vol. 77, no. 6, pp. 358-364, 2008.

[59] H. S. Akiskal, R. M. Hirschfeld, and B. I. Yerevanian, "The relationship of personality to affective disorders," Archives of General Psychiatry, vol. 40, no. 7, pp. 801-810, 1983.

[60] C. R. Cloninger, Feeling Good: The Science of Well-Being, Oxford University Press, New York, NY, USA, 2004. 


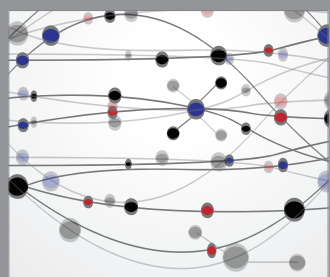

The Scientific World Journal
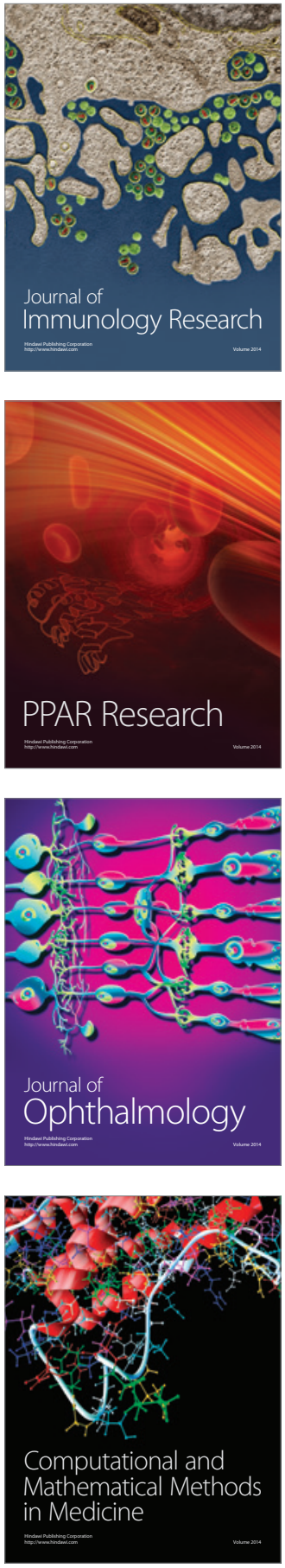

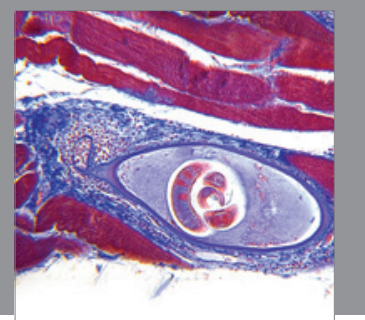

Gastroenterology

Research and Practice
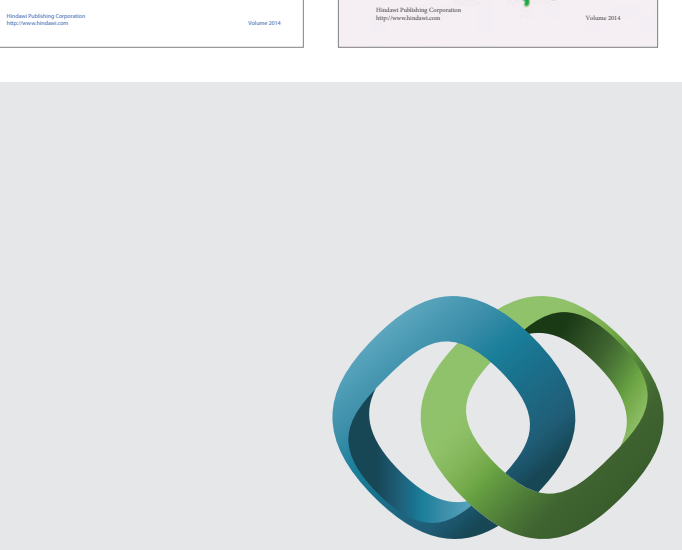

\section{Hindawi}

Submit your manuscripts at

http://www.hindawi.com
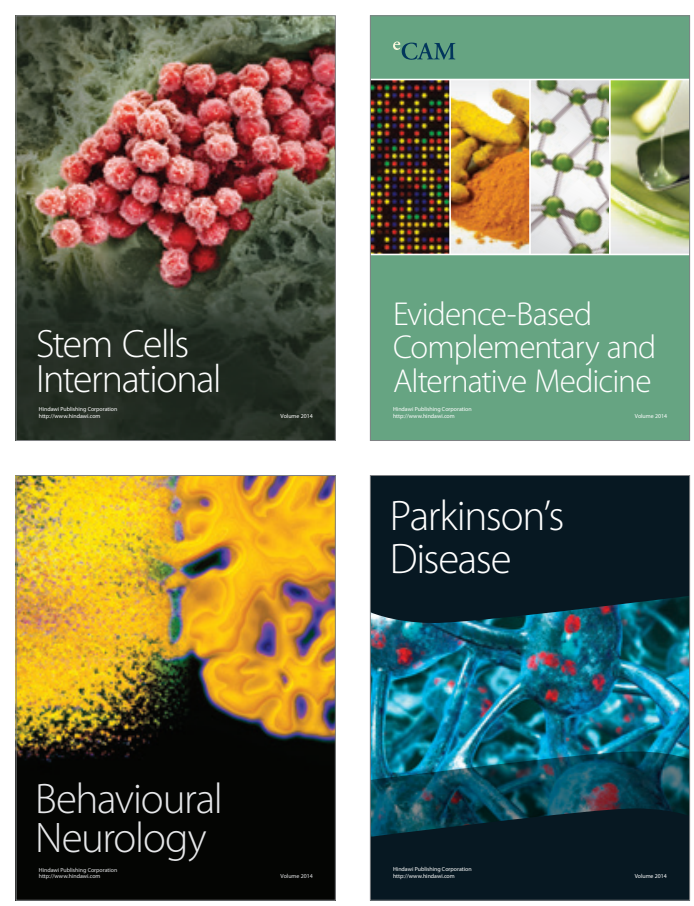

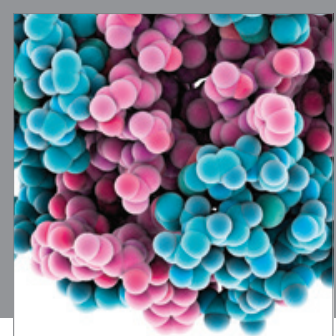

Journal of
Diabetes Research

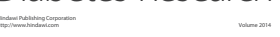

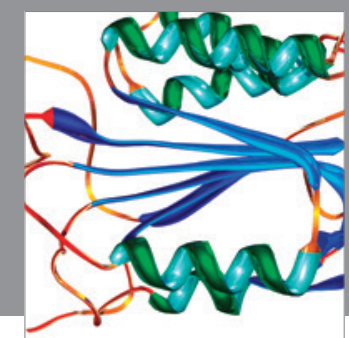

Disease Markers
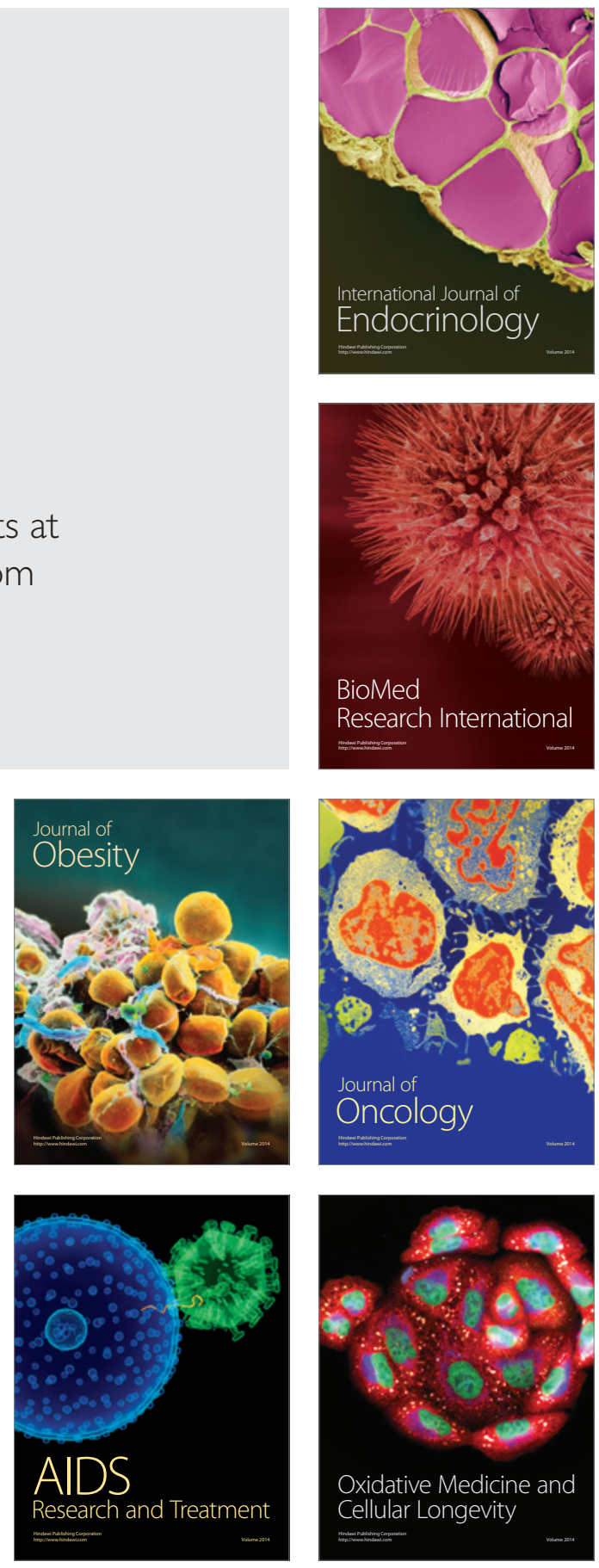